role in the domestication process. Their reasoning is simple - having scrutinized the Mesolithic and Neolithic bone record covering the preceding several millennia, they find no evidence that the wild camel, C. ferus, was present in these regions. Perforce, they argue, this cannot have been the area of original domestication, which they predict occurred farther to the east, perhaps in Kazakhstan, northern Mongolia or northern China, areas clearly inhabited by $C$. ferus. Even in China - despite the detailed understanding of camels revealed by the twelfth century $\mathrm{AD}$ medical manual - there is no direct evidence of the domestication process itself. The earliest surviving mention of Bactrian camels in the Chinese literature is as late as the fourth century $\mathrm{BC}$, long after the original domestication event.

How, then, did the human-camel relationship change from hunting to domestication? Contrary to their image as longdistance nomads, in the wild state, camels have a strong attachment to their home area. It is possible that, especially during droughts, camels and humans would have been brought into close contact, as both sought the same watering places. The authors postulate that it seems most likely that camels were first kept for their meat - they grow rapidly and can inhabit arid areas where the vegetation is insufficient to support cattle or sheep. Soon people learned to exploit the benefits of wool and dung - camels shed copious quantities of high-quality wool, especially in the spring moult, and their dung has excellent burning properties, producing a hotter, cleaner flame than wood (of value when fires are built in tents). Peters and von den Dreisch suggest that the use of camels for carrying heavy loads or as a means of transport came later.

This exemplary study of a neglected animal illustrates the rigorous approach necessary for appraising the origins of domestication in any species and, in so doing, demonstrates the maturity and importance of archaeozoology as a science.

Adrian M. Lister is in the Department of Biology,

University College London, London WC1E 6BT, UK. e-mail: a.lister@ucl.ac.uk

1. Franke, H. Sudhoffs Archiv. (in the press).

2. Peters, J. \& von den Dreisch, A. J. Zool. 242, 651-679 (1997).

\title{
Colour takes the field
}

\section{Frank Wilczek}

S ometimes discoveries in physics come as sudden revelations: the existence of a new kind of particle, or of a new phase of matter. But sometimes profound results take shape gradually, appearing first as suggestive but not unique interpretations of limited data, becoming fully realized only as they are recognized in different contexts and made quantitative. The phenomenon of colour coherence is of the latter type. Its observation ${ }^{1,2}$ is a very remarkable result, showing in a dramatic way the unity and predictive power of fundamental physical theories; yet it has come upon us so gradually that it has gone almost unremarked.

To set the stage, let me briefly recall the close analogies between quantum electrodynamics (QED) and quantum chromodynamics (QCD), the theory of the 'strong' force which binds atomic nuclei and governs most high-energy particle interactions. Both theories are based on the concept that fields respond to the presence and motion of charges. In QED, the electromagnetic field responds to electric charges. In QCD a more complicated system of eight different fields responds to the presence and motion of three different kinds of charge, somewhat inappropriately called colours ('red', 'green' and 'blue'). Although the mathematics of QCD is more intricate - and thus, to aficionados, more symmetrical, beautiful and challenging - than that of QED, the basic interactions postulated in the two theories are profoundly similar.

Electrons and positrons (among other particles) carry electric charge, and quarks and antiquarks are their colour counterparts. Photons are the corpuscles of the electromagnetic field of QED; the eight colour gluons are their analogues in QCD. Gluons are now routinely observed as individual particles, signalled by the jets of energetic particles they leave in their wake. But amid all the close analogies, there is one important difference between QED and QCD, which is responsible for their very different dynamics: whereas the photon is electrically

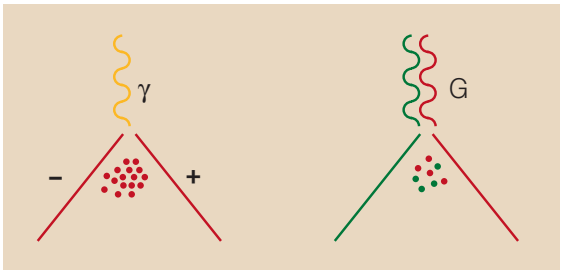

Figure 1 Addition of colours. These two types of three-jet event are produced by the annihilation of electrons and positrons. When a photon $(\gamma)$ accompanies a quark and antiquark, the quark and antiquark have equal and opposite colour charge (here, red). When there is a gluon $(G)$ instead, the quark and antiquark have different, but not opposite, colour charges. The coherently reinforced colour field in the first case creates far more particles than the incoherent fields in the second.

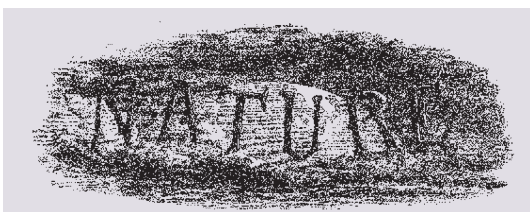

100 YEARS AGO

Different minds place different estimates on the intellectual accomplishments of the past half-century. In ordinary conversation the men of the mart will point to an Eiffel tower, a suspension bridge, a continental express train, a man-of-war, or an Atlantic cable. But in a discourse recently delivered in commemoration of the jubilee of the Sheffield Scientific School of Yale University, President Gilman remarked that perhaps the greatest triumphs of the intellect during the last half-century are these five contributions to human knowledge: the establishment of the principles of evolution; the establishment of the principle of the conservation of energy; the development of mathematical science and its application to physics, mechanics, electricity and astronomy; the development of spectrum analysis and the consequent discoveries respecting light and electricity; and the discovery of the nature and functions of bacteria, and of their influence, for weal or woe, upon living organisms.

From Nature 16 December 1897.

50 YEARS AGO

In 1914 Walter Jones began the preface to his monograph on nucleic acids with the words, "The nucleic acids constitute what is possibly the best understood field of Physiological Chemistry..." That was then a tenable point of view; but twenty years later it would have been absurd. There are fashions in everything, including biochemistry, and nucleic acids went largely out of fashion or were swamped by the growth of our knowledge of proteins, oxidation mechanisms and the other processes that characterized the development of biochemistry in the 1930's. The boom-slump-boom cycle is, however, not confined to economics, and a nucleic acid boom is now upon us. Much new knowledge of the intrinsic properties and biological behaviour of the nucleic acids has been gained during the past few years; but there seems to have been a disproportionate flood of review articles, conferences and symposia. Stocktaking is valuable, but what is now needed in cytochemistry is more stock rather than more surveying. N.W. Pirie

From Nature 20 December 1947. 
neutral, the gluons themselves carry colour charges (Fig.1).

The soul of QED is the electromagnetic field. When Faraday and Maxwell proposed this field, their contemporaries found it very abstract and mysterious. But as antennas were devised that danced to its tune, the electromagnetic field came to seem undeniably real and almost tangible.

Can one devise antennas for the colour fields of the strong interaction? It is not possible to do this directly, because the wavelengths involved are ridiculously small $10^{-14} \mathrm{~cm}$ and below, much smaller than Xrays or even ordinary gamma-rays. Fortunately, colour coherence provides an indirect path to the goal.

What follows is an example of colour coherence which makes an especially vivid impression, owing to its use of photons and gluons in contrast.

Usually, when a high-energy electron and its antiparticle, a positron, annihilate, two narrow sprays of particles (jets) emerge, produced by a short-lived quark-antiquark pair. Some slow-moving particles are also left behind. The number of particles produced at a given time and place measures the strength of the colour field there, much as an antenna's response measures the strength of electromagnetic fields. This multiplicity is roughly proportional to the intensity, or energy, of the field, which grows as the square of its magnitude. So, in effect, the slow-moving particles provide a peculiar form of antenna - one that is fairly crude, but unique in its sensitivity to exceedingly short-wavelength colour fields, ready-made and very cheap.

This antenna has been used to distinguish between coherent and incoherent fields, which are produced in two slightly rarer cases of electron-positron collision. In about ten per cent of the events, there is a third jet containing several strongly interacting particles. This third jet signals the radiation of a colour gluon. Still more rarely, in fewer than one per cent of the events, an energetic photon accompanies the quark and antiquark (Fig. 1). Fortunately, as millions of electron-positron annihilation events have been studied, there are many examples of both rare types available for analysis.

Colour charge, like electric charge, is conserved. Because the original electron and positron had zero colour charge, the colour charges of the annihilation products must add up to zero. When the quark and antiquark are accompanied by a photon, their colour charges must be equal and opposite, as the photon is colour neutral. The positive charge (red, say) creates a red colour field that points away from it, whereas the field of the negative red charge points towards it. In the region between, these two contributions reinforce each other - the fields are coherent. But when the quark and antiquark are accompanied by a gluon, their colour charges are generally of completely different types, not equal and opposite, and the fields between them do not reinforce each other.

QCD predicts, therefore, that more particles will be produced between the quark and antiquark jets when they are accompanied by a photon, as compared to when they are accompanied by a gluon jet. Just such an effect is observed. The difference is about a factor of two, which agrees quite well with rigorous theoretical calculations ${ }^{3}$.

Other, more complex colour coherence effects are seen in other types of event. For example, in collisions of particles other than electrons and positrons - such as quarks and antiquarks ${ }^{4}$, or quarks and electrons - the colour fields of the initial particles can reinforce, or cancel, the colour fields of the particles produced.

As these patterns are mapped out, colour fields are becoming an ever more tangible aspect of reality. The recent accumulation of definitive results from a variety of experiments makes this an appropriate time to declare a triumph.

Frank Wilczek is in the School of Natural Sciences,

Institute for Advanced Study, Olden Lane,

Princeton, New Jersey 08540, USA.

e-mail:wilczek@sns.ias.edu

1. Bartel, W. et al. Z. Phys. C 21, 37-52 (1983).

2. The ALEPH collaboration Phys. Rep. 24, 1-165 (1998).

3. Khoze, V. \& Ochs, W. Int. J. Mod. Phys. A 12, 2949-3120 (1997).

4. Abbot, B. et al. preprint hep-ex/9706012 on xxx.lanl.gov.

\section{Photonios}

\section{Frozen light}

\section{Sajeev John}

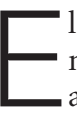
lectromagnetism is the fundamental mediator of all interactions in atomic physics and condensed-matter physics - in other words, the force that governs the structure of ordinary matter. It is rare to see an entirely new electromagnetic phenomenon, but Diederik Wiersma and colleagues report one on page 671 of this issue $^{1}$. In carefully prepared semiconductor
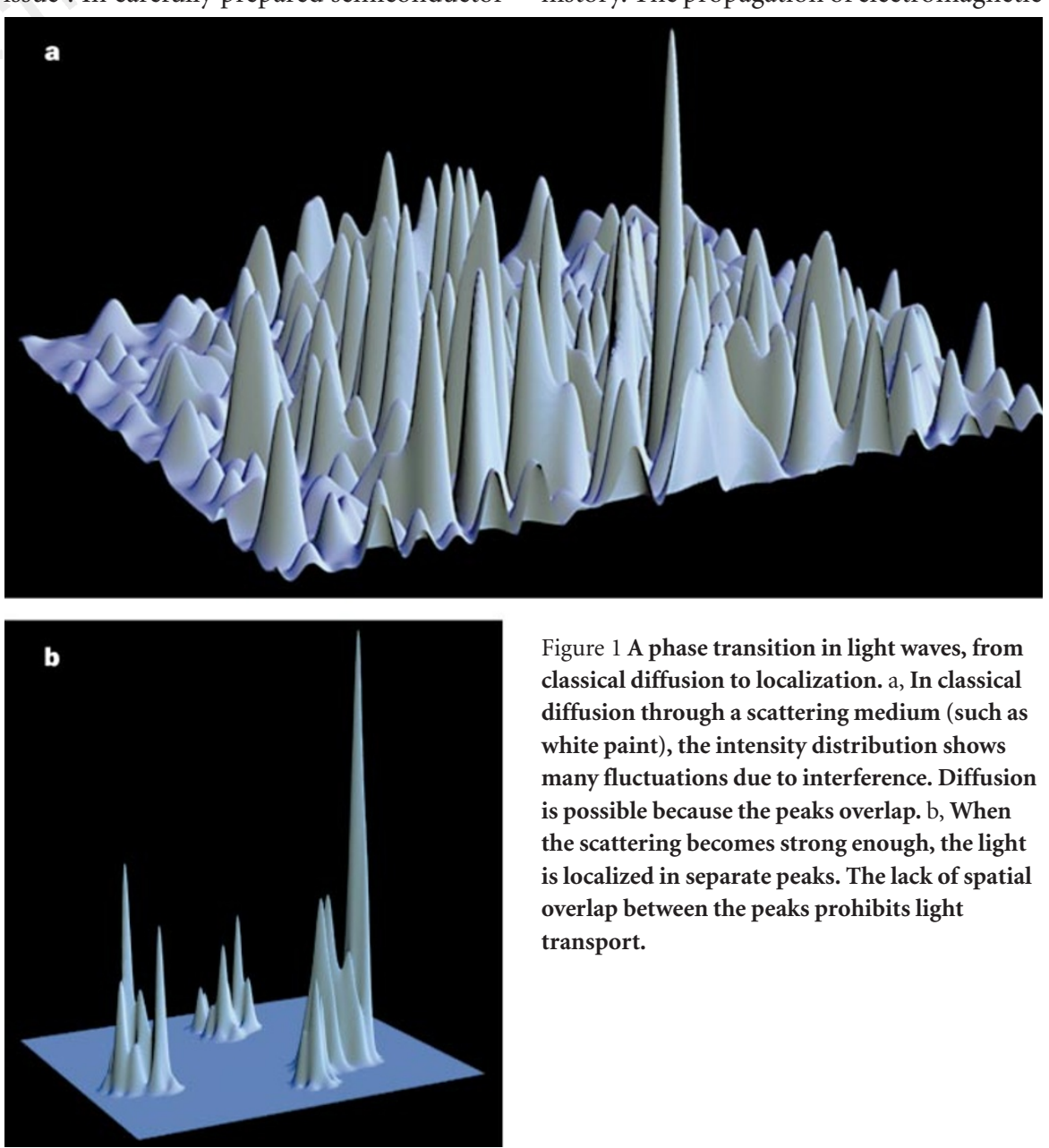

Figure 1 A phase transition in light waves, from classical diffusion to localization. a, In classical diffusion through a scattering medium (such as white paint), the intensity distribution shows many fluctuations due to interference. Diffusion is possible because the peaks overlap. $b$, When the scattering becomes strong enough, the light is localized in separate peaks. The lack of spatial overlap between the peaks prohibits light transport. 